\title{
RESEARCH ON SAFETY PROTECTION OF SMART MICROGRID OPERATION CONTROL BASED ON AUTOMATIC REGULATION
}

\author{
Guojun Shi \\ Heilongjiang Bayi Agricultural University, Daqing, \\ Heilongjiang 163319, China \\ Email: gushi72997624@163.com
}

\begin{abstract}
As a supplement to the growing power grid, the microgrid provides high-quality power services for users at the grid's edge. Maintaining the stability of the microgrid is conducive to providing users with high-quality power services and maintaining the safe operation of the grid. This paper briefly introduced the smart microgrid and the automatic regulation strategy used for controlling the stability of the microgrid, optimized the PI regulation strategy with the backpropagation (BP) algorithm, and improved the BP algorithm with particle swarm optimization (PSO). Finally, the traditional, the BP algorithm based, and the improved BP algorithm based PI regulation strategies were simulated in MATLAB software. The results showed that the three strategies could all make effective, stable regulation on the grid-connected voltage and frequency, but the improved BP algorithm based PI regulation strategy is more effective for grid-connected voltage and frequency control.
\end{abstract}

Keywords: Microgrid, Intelligent, Automatic Regulation, PI Control.

\section{Introduction}

For human production activities and daily life, efficient and clean energy is very important. Since the 21st century, electric power has gradually become the most commonly used energy in the whole society. Compared with traditional fossil energy, it is cleaner, more efficient, and safer in the use process, and more convenient in transmission [1]. To make power more fully used in people's life and production, the construction of the power grid has gradually become a major development foundation of China. Although the growing power grid improves the efficiency of power transportation, it also increases the management difficulty. In order to reduce the loss in the process of power transportation, high-voltage power transmission is usually used, which also brings risks to the security of the large-scale power grid [2]. The progress of technologies, such as the Internet, computer, and wireless communication and the market demand for the safe and efficient operation of large-scale power grids make smart grids emerge. A smart grid uses technologies, such as sensors, the Internet, wireless communication, and computer algorithm, to realize the automatic intelligent management of the power system. Deng et al. [3] proposed a multi-agent system-based smart microgrid that could improve power quality by dynamically and autonomously adjusting P-F and Q-U droop curves in local agents and verified the reliability of the method. Kumar et al. [4] proposed a coordinated control algorithm for distributed generators and direct current (DC) microgrids under islanding and grid-connected operation modes. The control strategy adopted a combination of feedback and feedforward control loops. The simulation results verified the robustness of the algorithm under different operating conditions, including fault scenarios and its effectiveness in maintaining the DC voltage of the microgrid. Acharya et al. [5] proposed an islanded microgrid voltage imbalance mitigation scheme by coordinating the photovoltaic grid-connected inverter and constant temperature control load, designed a negative sequence compensation loop that worked in parallel with the positive sequence compensation loop. They verified the effectiveness of the method through the simulation experiment. This paper briefly introduced the smart microgrid and the automatic regulation strategy that was used for controlling the stability of the microgrid, optimized the PI regulation strategy with the Back-Propagation (BP) algorithm, and improved the Back-Propagation (BP) algorithm with the particle swarm optimization (PSO). Finally, the traditional, the BP algorithm based, and the improved BP algorithm based PI regulation strategies were simulated on MATLAB software.

\section{Smart Microgrid and its Automatic Regulation}

Compared with the large-scale distribution network, the microgrid has relatively small output 
power. Moreover, the microgrid takes photovoltaic and wind power as power sources. This paper mainly focuses on photovoltaic power generation [6]. Although the scale of the microgrid is not as large as the distribution network, it is not so small that only one or two user loads participate in it. For the sake of illustration, the microgrid is simplified to some extent. For example, the complex user load in the microgrid is simplified as a single load, as shown in Figure 1. It was seen from Figure 1 that the basic structure of the microgrid includes a photovoltaic power supply, energy storage device, inverter, and load. The energy storage device is another source of power in the microgrid. Although photovoltaic power generation is clean and environmentally friendly, the output power fluctuation is relatively large limited by the light environment. The energy storage device can store the surplus power in the photovoltaic power generation process and supplement the photovoltaic power generation to stabilize the power output [7]. The energy storage device is also connected with the distribution network after being converted into alternating current power by an inverter. The load is the user that uses power in the microgrid. Although the scale of the microgrid is small, there is more than one user load. These users need different loads in different periods; therefore, users can be simply regarded as a load that can be adjusted randomly. In addition to the above basic structure, a smart microgrid also has a microgrid control center. Its function is to use sensors installed in the grid to collect power information and control the inverter in the microgrid after relevant algorithm processing to adjust the power output and ensure the stability of the power grid. The microgrid control center will also report the information collected by sensors to the superior general control center to obtain relevant feedback [8].

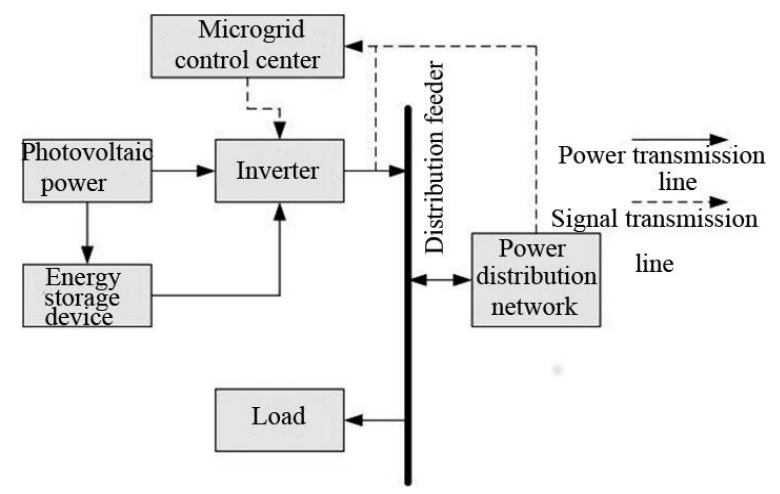

Figure 1: The Basic Structure of Smart Microgrid

To ensure power stability in smart microgrid operation, the microgrid control center will automatically adjust the inverter according to the power information collected by sensors. The control principle of the $P Q$ control strategy is shown in Figure 2. In Figure 2, $P$ and $Q$ refer to the actual active and reactive power respectively, and $P_{\text {ref }}$ and $Q_{\text {ref }}$ are the corresponding reference values. After the PI processing of the difference between the actual value and reference value, current reference values $i_{d, r e f}$ and $i_{q, r e f}$ of the corresponding $\mathrm{d}$ and $\mathrm{q}$ axes are obtained. Then, the reference values are compared with the actual grid-connected current of the corresponding $\mathrm{d}$ and $\mathrm{q}$ axes, i.e., $i_{d}$ and $i_{q}$. After the PI processing, the difference values were given coupling treatment along with the grid-connected voltage of $\mathrm{d}$ and $\mathrm{q}$ axes and the grid-connected current processed by $\omega L$ on the other axis to obtain the signal voltages of $d$ and $q$ axes for controlling the inverter, i.e., $U_{d}^{\prime}$ and $U_{q}^{\prime}$. After inverse park transformation for the signal voltage, the inverter is regulated by pulse-width modulation (PWM) [9].

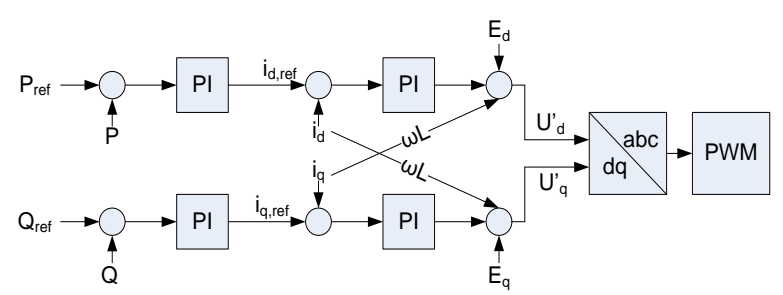

Figure 2: PQ Control Strategy of Smart Microgrid Under the Grid-connected Mode

\section{Automatic Regulation based on a Neural Algorithm}

\subsection{Combination of Traditional PI Regulation Strategy and BP Neural Algorithm}

The basic structure of a smart grid and PQ control strategy for automatic regulation of microgrid stability have been briefly described above. The PI control method is used to adjust the inverter output through error. The PI control method used in the microgrid control strategy in this study is the incremental digital PI control method [10], and the calculation formula is:

$$
u(k)=u(k-1)+k_{p}[e(k)-e(k-1)]+k_{i} e(k)
$$

where $u(k)$ and $u(k-1)$ are the control quantities for the control subject at the present time and last time, $e(k)$ and $e(k-1)$ are the error signals of the present time and the last time respectively, and $k_{p}$ and $k_{i}$ are proportional parameter and integral parameter, respectively.

In the traditional control strategy, $k_{p}$ and $k_{i}$ are fixed values, which are usually determined by working experience or field test, but it also makes the control strategy inflexible and ideal when controlling the large power grid. To make PI control 
adapt to the changing grid environment more flexible in the smart microgrid control strategy, the proportion and integral parameters are optimized by the neural algorithm so that they can change according to the changes of the grid environment.

In this study, the proportion and integral parameters are adjusted by the BP neural network algorithm. In this study, the data needed by the input layer of the BP neural network come from the voltage and current collected by the sensor at the grid connection, while the output layer outputs $K_{P}$ and $K_{I}$ of the transfer function in the controller. The hidden layer is the most important part of a neural network, and the activation function in the hidden layer can effectively fit the training data to obtain the hidden rules in the data. The basic steps of optimizing the parameters by the BP neural network are as follows. The grid data collected by sensors are input into the input layer. Then the input data are calculated layer by layer in the hidden layer. The output layer outputs the results. The results of the output layer are compared with the expected results.
The weight parameter in equation (2) is reversely adjusted according to the error [11]. The weight is constantly adjusted through repeated training until the training error reaches the expected value.

\subsection{Improving BP Algorithm with PSO}

As a machine algorithm, the BP neural network has an activation function that can approach the nonlinear law infinitely, which is very suitable for the changeable power grid environment. However, the BP neural network will over-fit in the training process, i.e., it will fall into the locally optimal solution. This problem can be solved by selecting training samples and initial parameters, but the neural network trained by specially selected training samples and initial parameters will be weak in universality. In addition to the above methods, this study improves the BP algorithm by reversely adjust parameters.

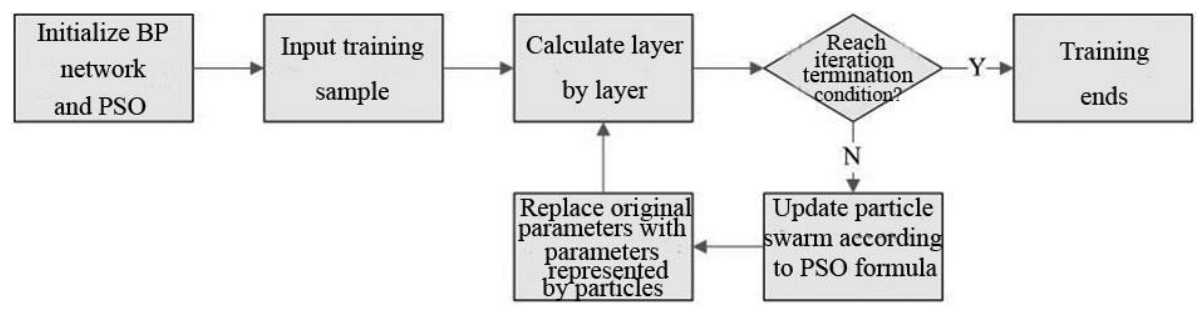

Figure 3: The Training Process of the Improved BP Neural Network

As shown in Figure 3, the steps of the improved algorithm in the forward calculation of the input data are the same as those of the traditional BP algorithm. After the forward calculation results are compared with the expected results, whether the error between them is in the expected range is determined. If not, the traditional BP algorithm adjusts the weight parameter reversely according to the error, but the improved BP algorithm adjusts the weight parameter through the PSO algorithm. Every particle in the PSO population represents a weight parameter scheme. The weight scheme in the population is updated according to the formula of PSO. The updating formula is:

$$
\left\{\begin{array}{l}
v_{i}(t+1)=\varpi v_{i}(t)+c_{1} r_{1}\left(P_{i}(t)-x_{i}(t)\right)+c_{2} r_{2}\left(G_{g}(t)-x_{i}(t)\right) \\
x_{i}(t+1)=x_{i}(t)+v_{i}(t+1)
\end{array}\right.
$$

where $v_{i}(t+1)$ and $x_{i}(t+1)$ are the speed and position of particle $i$ after one time of iteration, $v_{i}(t)$ and $x_{i}(t)$ are the speed and position of particle $i$ before iteration, $\varpi$ is the inertia weight of particles, $c_{1}$ and $c_{2}$ are learning factors, $r_{1}$ and $r_{2}$ are random numbers between 0 and $1, P_{i}(t)$ is the optimal position that particle $i$ has experienced, and $G_{g}(t)$ is the best position that the particle swarm has experienced. The original BP neural weight parameter is replaced by the updated population particle, and then the calculation is performed layer by layer again. The calculated results are compared with the expected results, and the difference is used as the fitness value of PSO. The above steps are repeated until the difference (fitness value) reached the set standard.

\section{Simulation Experiment}

\subsection{Experimental Environment}

In this study, the automatic regulation strategy in microgrid grid connection mode was simulated and analyzed using the MATLAB software [12]. The experiment was carried out in a laboratory server with configurations of Windows 7 operating system, 16 G memory, and Core i7 processor.

\subsection{Experimental Setup}

The basic structure of the microgrid used in the simulation experiment is shown in Figure 4. The 
microgrid structure adopted in this study also included the microgrid control center, which obtained the sensing information of the distribution network from the distribution feeder and output PWM control signal to adjust the inverter according to the built-in control strategy.

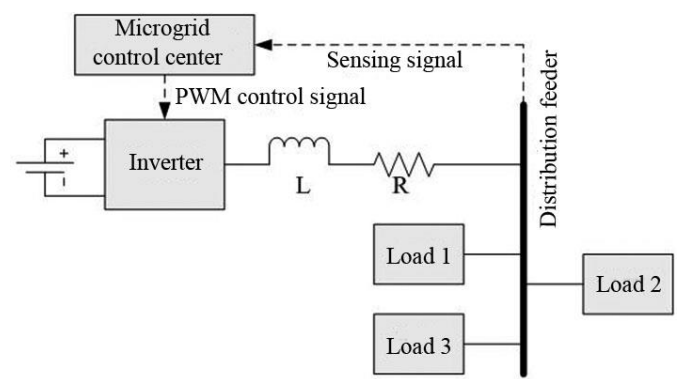

Figure 4: The Simulation Structure of Automatic Regulation Strategy in Microgrid Grid-connected Mode

The relevant parameters of the microgrid simulation model for the simulation experiment are shown in Table 1.

In addition to the above parameters, the relevant parameters of the automatic regulation strategy in the microgrid control center used for inverter control are as follows. (1).

When the control center adopted the traditional PI automatic regulation strategy, proportional parameter $k_{p}$ was 200, and integral parameter $k_{i}$ was 20. (2) When the control center adopted the BP algorithm based PI automatic regulation strategy, the proportion and integral parameters were adaptive parameters, which were determined by the $\mathrm{BP}$ algorithm; the initial weight in the BP algorithm was randomly generated between 0 and 0.5 [13]; the learning rate was 0.1 ; the maximum number of iterations was 1500. (3).

When the control center adopted the improved $\mathrm{BP}$ algorithm based PI automatic regulation strategy, the parameters needed by the BP algorithm were determined by PSO, and the parameters of PSO were as follows: the number of particle swarms was 30 , two learning factors were both set as 1.5, the maximum number of iterations was 1500 , and the inertia weight was 0.8 .

Table 1. Related Parameter Settings

\begin{tabular}{|l|l|l|l|}
\hline Parameter & Value & Parameter & Value \\
\hline $\begin{array}{l}\text { The rated voltage of the } \\
\text { microgrid }\end{array}$ & $220 \mathrm{~V}$ & $\begin{array}{l}\text { The rated frequency of the } \\
\text { microgrid }\end{array}$ & $50 \mathrm{~Hz}$ \\
\hline $\begin{array}{l}\text { The filter capacitance of } \\
\text { the filter }\end{array}$ & $20 \mu F$ & $\begin{array}{l}\text { The filter inductance of the } \\
\text { filter }\end{array}$ & $5 \mathrm{mH}$ \\
\hline $\begin{array}{l}\text { The filter resistance of } \\
\text { the filter }\end{array}$ & $0.1 \Omega$ & Load 1, 2, and 3 & $250 \Omega$ \\
\hline Line inductance & $0.5 \mathrm{mH}$ & Line resistance & $0.5 \Omega$ \\
\hline $\begin{array}{l}\text { The reference voltage of } \\
\text { the microgrid }\end{array}$ & $220 \mathrm{~V}$ & $\begin{array}{l}\text { The reference power of the } \\
\text { microgrid }\end{array}$ & $1 \mathrm{~kW}$ \\
\hline
\end{tabular}

\subsection{Experimental Project}

In brief, the experimental project aimed to control the stability of the microgrid by three automatic regulation strategies under the change of the load in the distribution network when the microgrid was connected to the grid. The specific steps are as follows: (1) the inverter is started and connected to the distribution feeder, and the three loads in the distribution feeder were also connected to the distribution network; (2) time started when the microgrid model started and the inverter and load were connected to the microgrid, load 3 was disconnected after running for $0.2 \mathrm{~s}$, and load 2 was disconnected after running for $0.4 \mathrm{~s}$.

The per-unit value, i.e., the relative value, was used in the calculation when the automatic regulation strategy of the microgrid control center was used. In this study, per-unit value = actual value collected by sensor /microgrid reference value.

\subsection{Experimental Results}

In this study, a working condition of gradually disconnecting the grid load was set up for the gridconnected microgrid under the three automatic regulation strategies to test the maintenance of the microgrid stability by the three automatic regulation strategies. The final results are shown in Figure 5. It was seen from Figure 5 that the grid-connected voltage was relatively stable under the three automatic regulation strategies. In the setting of the working condition, one load was disconnected at 0.2 $\mathrm{s}$, and the other at $0.4 \mathrm{~s}$. It was seen from Figure 5 that the voltage of the microgrid under the traditional PI automatic regulation strategy fluctuated significantly at the moment of load disconnection, with a fluctuation range of 1.03 1.38, the voltage fluctuation of the microgrid under the BP algorithm based PI automatic regulation strategy was smaller, about $1.02 \sim 1.16$, and lasted 
for a shorter time, and the voltage of the microgrid under the improved BP algorithm based automatic regulation strategy almost had no fluctuation and kept stable in the operation process.

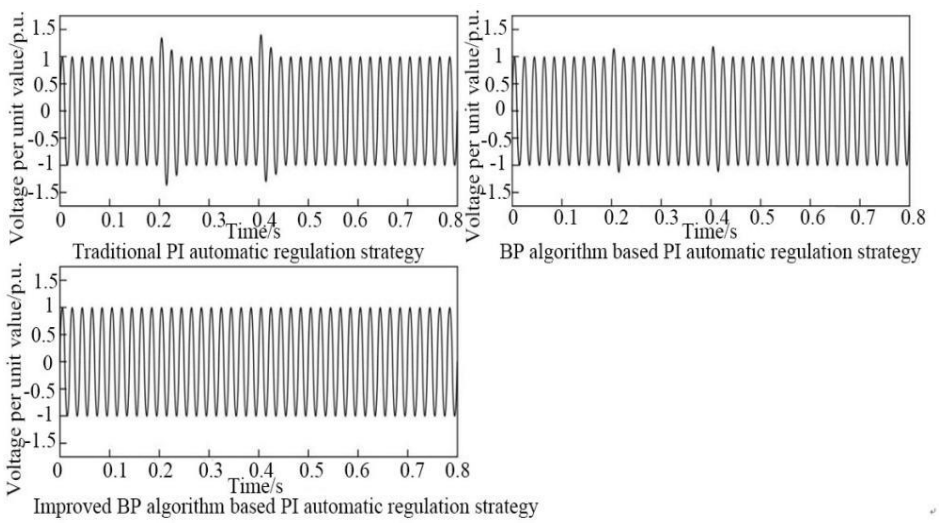

Figure 5: Variation of the Grid-connected Voltage of the Microgrid Under Three Automatic Regulation Strategies

In the set working condition, the stability test of the three automatic regulation strategies not only involved the above grid-connected voltage fluctuations but also included the frequency changes of the grid-connected power. The test results are shown in Figure 6. It was seen from Figure 6 that the power grid operating frequency under the three automatic regulation strategies fluctuated correspondingly in the face of load changes in the power grid. After operating for $0.2 \mathrm{~s}$, the frequency under the traditional PI regulation strategy, the BP algorithm based PI regulation strategy, and the improved BP algorithm based PI regulation algorithm fluctuated by about $0.03 \mathrm{~Hz}, 0.025 \mathrm{~Hz}$, and $0.007 \mathrm{~Hz}$, respectively.
After operating for $0.4 \mathrm{~s}$, the frequency under the traditional PI regulation strategy, the BP algorithm based PI regulation strategy, and the improved BP algorithm based PI regulation algorithm fluctuated by about $0.04 \mathrm{~Hz}, 0.03 \mathrm{~Hz}$, and $0.01 \mathrm{~Hz}$, respectively. In conclusion, the frequency of the three strategies was almost stable under automatic regulation. Even for the traditional PI regulation strategy, the maximum frequency fluctuation was only $0.05 \mathrm{~Hz}$, which was not significant in the grid-connected voltage.

The comparison of the three strategies suggested that the improved BP algorithm based PI regulation strategy had a smaller frequency fluctuation.

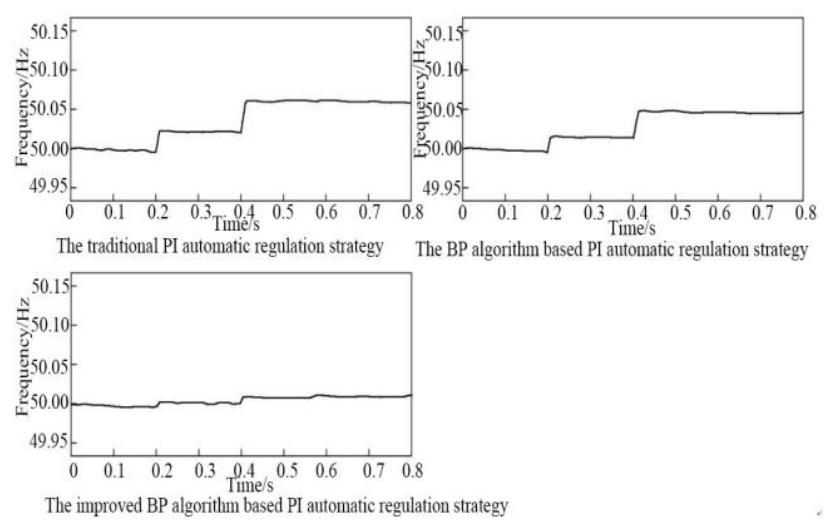

Figure 6: Variation of Microgrid Grid-connection Frequency Under Three Automatic Regulation Strategies

\section{Conclusion}

This paper briefly introduced the smart microgrid and the automatic regulation strategy that was used for controlling the stability of the microgrid. The PI regulation strategy was optimized by the BP algorithm, and the BP algorithm was improved by PSO. Finally, the traditional, the BP algorithm based, and the improved BP algorithm based PI regulation strategies were simulated in the MATLAB software.
The results are as follows. In the face of the change of load in the power grid, the three regulation strategies could maintain the stability of the gridconnected voltage, but the stability of the gridconnected voltage was the best under the improved BP algorithm based PI regulation strategy; when the load fluctuated, the voltage almost had no change under the improved BP algorithm based strategy, followed by the BP algorithm PI regulation strategy and the traditional PI regulation strategy. In the face of load changes in the power grid, the frequency fluctuation under the improved BP algorithm based 
PI regulation strategy was the smallest at the moment of load fluctuation, while the frequency fluctuation under the traditional PI regulation strategy was the largest.

\section{References}

[1] Sharma D, Dubey A, Mishra S, Mallik R K. "A Frequency Control Strategy Using Power Line Communication in a Smart Microgrid," IEEE Access, 2019, 7: 21712-21721.

[2] Carli R, Dotoli M, Jantzen J, Kristensen M, Othman $\mathrm{S}$ B. "Energy scheduling of a smart microgrid with shared photovoltaic panels and storage: The case of the Ballen marina in Sams," Energy, 2020, 198(May1):117188.1-117188.16.

[3] Deng C, Chen Y, Tan J, Xia P, Liang N, Yao W, Zhang Y. "Distributed Variable Droop Curve Control Strategies in Smart Microgrid," Energies, 2017, 11(1):24.

[4] Kumar M, Srivastava S C, Singh S N. "Control Strategies of a DC Microgrid for Grid Connected and Islanded Operations," IEEE Transactions on Smart Grid, 2017, 6(4):1588-1601.

[5] Acharya S, El-Moursi M S, Al-Hinai A, et al. "A Control Strategy for Voltage Unbalance Mitigation in an Islanded Microgrid Considering Demand Side Management Capability," IEEE Transactions on Smart Grid, 2019, 10(3):2558-2568.

[6] Liu Y T, Lai C M. "LCL Filter Design with EMI Noise Consideration for Grid-Connected Inverter," Energies, 2018, 11(7):1646-.
[7] Yang T, Gui W. "Research on multi-objective coordinated control strategies of grid-connected inverter under unbalanced voltage conditions," Transactions of China Electrotechnical Society, 2015, 30(11):148-157.

[8] Wu F, Sun B, Duan J, Zhao K. "Online Variable Topology-Type Photovoltaic Grid-Connected Inverter," IEEE Transactions on Industrial Electronics, 2015, 62(8):4814-4822.

[9] Wu F, Li X, Feng F, Gooi H B. "Modified Cascaded Multilevel Grid-Connected Inverter to Enhance European Efficiency and Several Extended Topologies," IEEE Transactions on Industrial Informatics, 2017, 11(6):1358-1365.

[10] Zheng X, Xiao L, Lei Y, Wang Z. “Optimisation of LCL filter based on closed-loop total harmonic distortion calculation model of the gridconnected inverter," IET Power Electronics, 2015, 8(6):860-868.

[11] Ozsoy E, Padmanaban S, Mihet-Popa L, Fedák V, Ahmad F, Akhtar R, Sabanovic A. "Control Strategy for a Grid-Connected Inverter under Unbalanced Network Conditions-A Disturbance Observer-Based Decoupled Current Approach," Energies, 2017, 10(7):1067-.

[12] Dai Y, Zhuang S, Li W, Luhang J J. “Dual-buck full-bridge grid-connected inverter with common-mode leakage current elimination," Electronics Letters, 2018, 54(2):93-95.

[13] Lo K Y, Chen Y M, Chang Y R. "Bidirectional Single-Stage Grid-Connected Inverter for a Battery Energy Storage System," IEEE Transactions on Industrial Electronics, 2017, 64(6):4581-4590. 